\title{
INTRODUCTION: A SOCIOPOLITICAL PERSPECTIVE
}

\section{Outline}

Introduction

What Is a Socially Just Psychology?

Why Should Social Justice Matter?

Can Social Justice Be Incorporated into Practice?

Should Social Justice Be Incorporated into Practice?

Taking Up the Challenge

Ethical and Professional Responsibilities

Structure and Overview of the Book

Conclusion

Discussion Questions

Web Links

Key Terms

\section{Introduction}

The past I5 years have witnessed significant and far-reaching developments in counseling about the topic of diversity. Studies on multiculturalism have attended very clearly to ways that diversity exists and interacts within individuals as well as conceptual and practical ways to attend to this diversity. The attention to ethnicity, class, and gender as important contributors to personal identity sensitizes counselors and enables them to recognize and incorporate this awareness into their practice with clients. While culturally sensitive practice has contributed to increased awareness by counselors of their own culture and identity as well as the culture and identities of their 
clients, the focus has remained on lateral similarities and differences across individuals and cultures.

Within multicultural counseling calls for social justice have been made. These calls necessitate awareness of not only lateral diversity through respect for and attention to cultural difference but also hierarchical diversity through recognition and attention to oppression and liberation. From such a perspective there are different experiences of power associated with membership in and identification with different cultural groups. The ways to attend to and incorporate this understanding into practice require counselors to reconsider how their own and others' social and political experiences factor in to perceived causes and appropriate interventions.

In this chapter, the concept of social justice is presented to reflect calls from within the profession for counselors to develop this understanding and consider approaches to their work that are informed by it. A brief description and history of social justice are followed by an overview of the contents of this book. The chapters that follow include ideas about counseling practice that are not only sensitive to oppression, but that also suggest how oppression may be indirectly and directly challenged by counselors within and outside of their offices.

Social justice has been suggested as the fifth force in counseling (Pieterse, Evans, Risner-Butner, Collins, \& Mason, 2009). A social justice approach to counseling "refers to using all of the methods of counseling and psychology to confront injustice and inequality in society" (Kiselica \& Robinson, 200I, p. 387). However, efforts to address injustice and inequality are not new to the profession. Indeed, the origins of career counseling, a precursor to contemporary counseling, were rooted in vocational guidance. The founder of vocational guidance, Frank Parsons, noted in I909 that power and money were unevenly distributed in the quickly changing industrial landscape. In addition to his work through the Vocational Bureau in Boston, Parsons advocated for youth and women as well as those without financial means or housing (Hartung \& Blustein, 2002).

Counseling has a social justice orientation but its expression was supplanted by the major theoretical developments influencing professional practice. The first of these forces was psychoanalysis with its emphasis on unconscious and intrapsychic determinates of psychopathology. The second force was cognitive with its emphasis on operational and contingent determinates of behavior. The third force was humanistic with its emphases on individuals' acceptance and expressions of freedom and responsibility. Attention to the social environment and relationship to intrapsychic processes became more prominent with the fourth force of psychotherapy, multicultural counseling.

During the I970s cultural diversity began to appear as a topic at conferences and in the professional literature, reflecting dissatisfaction with counselors' abilities to address the needs of an increasingly diverse population. The American 
Psychological Association formed a task force to develop multicultural competencies, which led to the production of the first document by Derald Wing Sue and colleagues in 1982. The most recent version of these competencies was approved in 2002 by the American Psychological Association's Council of Representatives and is known as the Guidelines on Multicultural Education, Training, Research, Practice, and Organizational Change for Psychologists.

Feminist and critical views have made major contributions that brought attention to the interrelated social and political conditions that produce disadvantage. There is growing support within counseling for practice that is sensitive and responsive to diversity. However, there has been less attention given to the use and liberating effects of culturally based psychotherapies. Additionally, the recognition of forces outside of the individual as legitimate targets of intervention by counselors has not yet been connected with actionable ideas or strategies. This book provides some ways to reconsider sociopolitical assumptions upon which counseling practice is often based and to apply social justice in counseling.

\section{What Is a Socially Just Psychology?}

Contextual factors in culturally competent counseling practice have been attended to. In the US, Sue and Sue's (2003) influential thinking on the subject has attended to individual, professional, organizational, and societal levels of intervention. In Canada, Arthur and Collins (20I5) have identified the need for action by counselors to promote change to systems that negatively affect their clients. What is missing is a comprehensive account of the types and nature of sociopolitical forces, their interactions and impact on individuals and communities.

A basic premise of this book is that: (a) sociopolitical forces operate and interact with individuals in ways that (b) produce inequities, which (c) create conditions of oppression based on cultural group membership, and (d) diminish their potential for and experience of well-being. Social justice can be considered a deliberate effort through both intrapersonal and community interventions "to minimize oppression and injustice in favor of equality, accessibility, and optimal developmental opportunities for all members of society" (Kennedy \& Arthur, 20I4, p. 188).

\section{Why Should Social Justice Matter?}

Health status varies considerably based on group membership. In health care, life expectancy (Statistics Canada, 20I6), self-reported health status (Alang, McCreedy, \& McAlpine, 20I5), and reporting of health problems (Veenstra \& Patterson, 20I6) each vary according to cultural group membership. 
In relation to mental health, residence in the most economically challenged communities is associated with the highest levels of mental health need, but the lowest access to and utilization of services (Durbin, Moineddin, Lin, Steele, \& Glazier, 2015).

Unearned privilege exists and offers several advantages (Muntaner, $\mathrm{Ng}$, Vanroelen, Christ, \& Eaton, 20I3). The conditions into which we are born have a significant influence on how long we will live, our material circumstances, and our quality of life. Said another way, the playing field of life is unequal. The consequences of this unequal playing field tend to reproduce themselves (Porter, 2015). For example, families with high income and educational status tend to have offspring who enjoy similar advantages in their own adult lives. In order to increase opportunities for those experiencing the most disadvantage, alteration of the field itself offers potential for meaningful change.

Counseling helps individuals cope with their challenging circumstances. It can enhance intrapersonal strength to the levels at which resiliency can be activated. For some, this makes it possible to rise against conditions that oppress and move toward a more favorable set of conditions. Professionals can simultaneously promote wellness among individuals and groups through efforts to challenge oppressive sociopolitical forces. Finally, there is a substantial interest in the concept and application of social justice and social action among graduate students and faculty (Ali, Liu, Mahmood, \& Arguello, 2008; Baluch, Pieterse, \& Bolden, 2004; Beer, Spanierman, Greene, \& Todd, 2012; Brown, Collins, \& Arthur, 20r4; Constantine, Hage, Kindaichi, \& Bryant, 2007; Goodman, Liang, Helms, Latta, Sparks, \& Weintraub, 2004; Miller \& Sendrowitz, 20ı ; Palmer \& Parish, 2008; Pieterse, Evans, Risner-Butner, Collins, \& Mason, 2009; Singh, Hofsess, Boyer, Kwong, Lau, McLain, \& Haggins, 20IO).

\section{Can Social Justice Be Incorporated into Practice?}

A significant barrier to the implementation of social justice in counseling is identifying what it is and how to do it. It is important to distinguish social justice conceptually from multicultural counseling. Multicultural counseling attends to notions of inclusion and acceptance of diversity, while social justice focuses on oppression and the marginalization that occurs as a consequence of it (Pieterse, Evans, Risner-Butner, Collins, \& Mason, 2009). Relatively little attention has been paid to the conceptual and practical application of social justice for counselors (Winter \& Hanley, 2015). It has been noted (Pieterse, Evans, Risner-Butner, Collins, \& Mason, 2009) that "if counsellors and counseling psychologists are indeed seeking to accept social justice as a central aspect of their training and identity, current efforts to address social 
justice at the level of counseling psychology and training may be inadequate or not clearly articulated" (p. I09).

Fortunately, there are models that can be drawn from that include underlying principles, counselor development, and outcomes of social justice. Based on multicultural, feminist, and critical psychology, underlying principles have been identified (Goodman, Liang, Helms, Latta, Sparks, \& Weintraub, 2004). These principles include: (a) self-examination, (b) sharing power, (c) giving voice, (d) raising consciousness, (e) using a strengths focus, and (f) providing tools for personal and social change. Requisites for this work are located in (a) self-awareness, (b) awareness of client, (c) integration of this awareness into working alliances, and (d) social action to influence systems (Arthur \& Collins, 20I5). Finally, specific efforts associated with this awareness by counselors may include: (a) challenging oppression, (b) empowering communities, (c) engaging in political advocacy, as well as (d) promoting a social justice agenda in professional associations (Palmer \& Parish, 2008).

\section{Should Social Justice Be Incorporated into Practice?}

Some professionals are reluctant to engage in social action, and for good reason (Helms, 2003). First, efforts on behalf of or in partnership with disadvantaged communities are rarely funded. The reality is that many counselors have debt after years of study, and all have bills to pay. Second, community and political action threaten the perception by some of the "value free" status of the profession. However, as will be considered later in this book, counseling and its professional organizations are neither value neutral nor disconnected from the sociopolitical forces in play. Third, those who associate community action with ideas and activists on the political left may reject it as too radical. Fourth, social justice takes a great deal of time and energy with unpredictable results. Such efforts can be tiring and take energy away from pursuits that offer needed balance in a demanding line of work. Finally, such efforts can raise the profile of counselors who may be viewed as critical of colleagues, employers, or funders which could threaten their professional practice.

\section{Taking Up the Challenge}

From a social justice perspective, however, the ways counselors view themselves, clients, and communities, as well as their roles and responsibilities in relation to each, may be challenged. For example, who really benefits from our work? Does our work make a difference where a difference really matters (Miller \& Sendrowitz, 20II)? This perspective also pressures counselors to recognize themselves as cultural people who experience privilege and oppression and occupy 
different positions on the unequal playing field. Once counselors have engaged in that work, they can more readily recognize the cultural identities, lives, and experiences of clients as both unique as well as similar and different from their own.

A socially just counseling practice is based in the counselor's abilities to recognize, engage, and connect through similarity and across difference. It also requires service through therapeutic as well as external work. Perhaps the most challenging aspect of a social justice perspective is to consider how interrelated the personal and professional lives of counselors are and how they may contribute to an unjust status quo. In addition, there is a challenge to be politically conscious and deliberate in both social action and inaction.

\section{Ethical and Professional Responsibilities}

Conflicting opinions exist about the evidence in support of social justice within the major codes of ethics. However, the American Psychological Association and the Canadian Psychological Association reflect awareness and commitment to social justice, as evidenced by their principles of equity, access, participation, and harmony. There is, however, no commitment to social action (Winter, 20I5).

Professionally, the topic of justice has been a focus of attention and Prilleltensky (20I3) offers a convincing argument in support of the need for fairness to achieve wellness. While the societies in which we live focus on merit to promote fairness, it is argued that because of the unequal playing field, the principle of need should be favored. Prilleltensky goes on to argue that individuals, communities, and societies can and should have maximum wellness that does not come at another's expense. A worthy ethical and professional pursuit is to learn to practice in a multicultural, multiethnic, multi-faith, multi-sexual, multi-racial, multi-gendered, and multi-abled society (Moodley \& Palmer, 20I4; Moodley \& Kinha, 2015).

In this book, readers are invited to consider their practice with individuals experiencing oppression as well as ways they might engage in external work to target sociopolitical forces that both disadvantage and advantage cultural groups.

\section{Structure and Overview of the Book}

This book is divided into two sections: "Understanding the Context" and "Changing the Context." In the first section, an argument in support of a sociopolitical view of mental health and professional counseling practice is made. In the second section, ways that professional counselors can support community level change are offered. 
"Understanding the Context," Chapters I-4, begins with an introduction to sociopolitical forces and their relationship to personal wellness. Attention is then drawn to personal and professional values and beliefs associated with the topics of oppression and liberation. Finally, ways that oppression and liberation may be attended to in counseling practice are considered.

In Chapter I, the focus is on recognition that the training counselors receive is contextually situated. A focus on intrapersonal change through altering perspective or interpreting present or early life experiences and thoughts or behaviors often does not recognize the sociopolitical context within which it occurs. This sociopolitical context offers great potential for considering forces that contribute to wellness of communities and individuals.

In Chapter 2, the emphasis is on the sociopolitical nature of mental health. Evidence concerning population trends in wellness are juxtaposed against the limitations of institutionalized medicine that narrowly define health as illness to prescribe treatment for symptoms. The range of contributors to wellness offers areas within which change that affects many can be made. Because sociopolitical disadvantage at the population level is associated with psychological effects at the individual level, a psychological perspective on oppression and liberation is offered.

In Chapter 3, attention is turned to the counselor. The values and beliefs that guide the practice of counselors are a mixture of political, professional, and personal. Counselors who are aware of the experiences they have in relation to privilege and oppression, similarity and difference, as well as inclusion and exclusion can take deliberate actions in accordance with their views. Views about society and its citizens, professions and professionals, as well as the roles counselors fill contribute either to sociopolitical change or maintaining the status quo.

In Chapter 4, attention is focused on culturally based psychologies and their contributions to wellness. Counseling practice in a sociopolitical context may itself be oppressive when it reinforces dominant views concerning causes and interventions for mental health on an individual who identifies with and experiences life from a non-dominant cultural worldview. Cultural psychologies are clearly located in specific worldviews that may be appropriate for understanding and working sensitively and effectively with clients who identify with them.

"Changing the Context," Chapters 5-ro, begins with a conceptual model that links sociopolitical awareness to community change. Forces that impact communities are considered before a review of major approaches, applications, and tactics of community change. A model for community change is offered as a way for counselors to identify processes and determine their desired level and type of involvement. Specific examples of community change are presented 
and followed by a distillation of principles that demonstrate their application within organizations and programs. Finally, considerations for evaluation of community change efforts are presented.

In Chapter 5, a conceptual model is presented that incorporates sociopolitical forces of oppression and liberation with individual psychological experience via cultural identity. The purpose of the model is to link individual, personal psychological well-being with sociopolitical determinates of health. The concept of intersectionality is invoked to explain how cultural identities can be claimed by an individual as well as represent collective experiences of group members. In this model, individual and community wellness share a common basis from which interventions can follow.

In Chapter 6, the focus is on community as a target of intervention. Major sociopolitical forces that affect communities are presented to highlight contributors and detractors from collective empowerment. The concept of social capital is used to characterize interrelationships between members that represent a source of power. Power is suggested to be a force that operates to maintain the status quo as well as create change.

In Chapter 7, the emphasis is conceptual, concerning ways that community change can be pursued. The efforts of leaders in community change are profiled to illustrate different approaches, such as development, planning, and action. The benefits and drawbacks of each are presented as are the conditions under which each is favored. Current models of practice for community change offer a range of possibilities for incorporating one or more approaches for collective benefit. Specific tactics are also presented.

In Chapter 8, the focus is practical and includes steps that could be followed to promote community change. It is emphasized that counselors armed with this understanding about a process of community change can be deliberate in their level of participation at different phases of the process. For each type of participation, the potential benefits and drawbacks are considered. Ethical issues that could be encountered by counselors involved in community work are also identified.

In Chapter 9, examples of community change efforts are presented. The types of change range from a single issue and single approach to multiple related issues and approaches. The application of community change processes and skills can be extended to work within organizations and in program development. The same principles of community-based, asset-oriented approaches that are grounded in cultural awareness and appropriateness apply to work outside of organizations, organization building, organizational change, as well as the development of new initiatives and group-based services.

In Chapter Io, the topic of evaluation is considered. Change at community levels, organizations, and programs, as with change experienced by individuals in counseling, can be similarly assessed. The scope, method, and topic varies 
according to the purpose, needs, and resources of the evaluator. However, similar principles apply.

\section{Conclusion}

Social justice instructs practitioners to attend to the personal effects of social hierarchies produced by inequality. From such a view, counseling contributes to the creation and promotion of equal opportunities for participation by all citizens of a society. It is argued that without social change, limits on personal change for those who are most disadvantaged are neither adequate nor sustainable. As well, without a view to social justice, professional efforts may actually preserve the status quo and reinforce inequitable social positions.

\section{Discussion Questions}

I. What is an appropriate basis of a "just society"? Consider the ideas of both merit (benefits based on performance) and need (benefits based on opportunity). In a just society, what is the fairest basis for decision making and outcomes?

2. Social justice has a long history outside of psychology. What do you think of when you read about social justice? Is your reaction to the idea positive, negative, or mixed? Does it have any place in the counseling profession? Why or why not?

3. Are all problems that are brought to the attention of a counselor the result of living in an unjust society? Why or why not?

4. Counselors have a primary ethical and professional responsibility to the clients they work with. What should counselors do when they and their client locate the source of his or her problem as outside of himself or herself?

\section{Web Links}

- Counselors for Social Justice (American Counseling Association) https:// counseling-csj.org

- Counsellors for Social Justice (Canadian Counselling and Psychotherapy Association) https://www.ccpa-accp.ca/Chapters/social-justice/

- Women and Psychology Section (Canadian Psychological Association) 
http://www.cpa.ca/cpasite/UserFiles/Documents/publications/ guidelines\%2ofor\%2opsychological\%2opractice\%20women.pdf

- Aboriginal Psychology Section (Canadian Psychological Association) http://www.cpa.ca/aboutcpa/cpasections/aboriginalpsychology

- The Society for the Psychological Study of Social Issues (American Psychological Association)

https://www.spssi.org/index.cfm?fuseaction=Page.viewPage\&pageId= 483 \&parentID $=479$

Key Terms

fifth force

first force

second force

third force fourth force

multicultural competencies

social justice

social action 